\title{
Efecto de miconazol sobre el recuento de levaduras en candidiasis asociada a estomatitis protésica.
}

\section{Effect of miconazole on the yeast count in candidiasis associated with denture stomatitis.}

\author{
Blanca Urzúa-Orellana' ${ }^{1}$, Patricia Palma-Fluxá ${ }^{2}$, Juana Olga Salinas-Flores ${ }^{3}$, \\ Ximena Lee-Muñoz ${ }^{4}$,Andrea Cortés-Coloma ${ }^{1}$, Cristian Vergara-Núñez ${ }^{5}$, \\ Ana Ortega-Pinto ${ }^{2}$, Iris Espinoza-Santander ${ }^{2}$, Irene Morales-Bozo ${ }^{1 *}$
}

1. Instituto de Investigación en Ciencias Odontológicas, Facultad de Odontología, Universidad de Chile. Santiago, Chile.

2. Departamento de Patología y Medicina Oral. Facultad de Odontología, Universidad de Chile. Santiago, Chile.

3. Centro de Referencia de Salud Cordillera Oriente. Servicio de Salud Metropolitano 4. Departamento de Prótesis. Facultad de Odontología, Universidad de Chile. Santiago, Chile. 5. Departamento del Niño y Ortopedia Dentomaxilar. Facultad de Odontología, Universidad de Chile. Santiago, Chile.

${ }^{*}$ Correspondencia autor: Irene Cecilia MoralesBozo | Dirección: Sergio Livingstone Pohlhammer 943, Independencia, Santiago, Chile. |

Teléfono:+56-2-9781792 | Email: imorales@ odontologia.uchile.cl

Trabajo recibido el 20/11/2017. Aprobado para su publicación el 15/04/2018

\section{RESUMEN}

Introducción: Estomatitis Subprotésica, proceso inflamatorio crónico de la mucosa adyacente a prótesis removible. $71,4 \%$ de los sujetos con esta condición es portador de Candida y la severidad se relaciona con la presencia de esta levadura. Para su tratamiento se indica antimicóticos tópicos de la familia de polienos o de azoles. El propósito del estudio fue determinar el recuento de levaduras del género Candida en adultos mayores con candidiasis oral, antes y después de ser tratados con miconazol. Materiales y métodos: Se consignaron antecedentes sistémicos y locales en 32 adultos mayores con estomatitis subprotésica. Se determinó recuento de levaduras del género Candida en saliva, antes y después del tratamiento tópico con Miconazol $2 \%$. Se aceptaron diferencias estadísticamente significativas con un error alfa igual o menor a $0.05 \%$. Resultados: Los recuentos de levaduras del inicio del estudio disminuyeron significativamente a los días 8 y 15 después del tratamiento (mediana 6.800, 163, 60, respectivamente). $56,2 \%$ de los individuos presentó persistencia de levaduras después del tratamiento; $21,8 \%$ de ellos con recuentos superiores a $400 \mathrm{UFC} / \mathrm{ml}$ de saliva. Conclusiones: En el $56,2 \%$ de los individuos del estudio se observó persistencia de levaduras del género Candida luego de 2 semanas de tratamiento con miconazol al $2 \%$.

PALABRAS CLAVE

Estomatitis subprotésica, Levaduras, Candida, Miconazol.

Rev. Clin. Periodoncia Implantol. Rehabil. Oral Vol. 11(2); 102-105, 2018.

\section{ABSTRACT}

Introduction: Denture stomatitis is a chronic inflammatory process of the mucosa adjacent to removable prosthesis. $71.4 \%$ of the subjects with this condition are carriersof Candida and the severity is related to the presence of this yeast. Topical antimycotics belonging to the polyene or azole family are indicated for its treatment. Efficacy of miconazole is reported to be from $80 \%$ to $100 \%$, although resistance is described in isolates of Candida. The purposeof the study was to determine the count of Candida in older adults with oral candidiasis,before and after being treated with miconazole. Methodology: Systemic and localantecedents were recorded in 32 elderly adults with denture stomatitis. Differences in number of the colony forming units of Candida yeast were determined before and after topical treatment with Miconazole $2 \%$. Statistical significances were set at a value of $p<0.05$. Results: Yeast counts at the start of the study significantly decreased 8 and 15 days after treatment (median 6,800, 163, 60, respectively). $56.2 \%$ of the subjectspresented persistence of yeasts after treatment; $21.8 \%$ of them with counts higherthan $400 \mathrm{CFU} / \mathrm{ml}$ saliva. Conclusion: In $56.2 \%$ of the study subjects, persistence of Candida yeasts was observed after 2 weeks of treatment with $2 \%$ miconazole.

\section{KEYWORDS}

Denture stomatitis, Yeast, Candida, Miconazole.

Rev. Clin. Periodoncia Implantol. Rehabil. Oral Vol. 11(2); 102-105, 2018. 


\section{INTRODUCCIÓN}

La Estomatitis Sub Protésica (ESP) es un proceso inflamatorio crónico de la mucosa adyacente a la prótesis removible, generalmente asintomático y cuyo diagnóstico es fundamentalmente clínico.

La etiología multifactorial de ESP, identifica factores predisponentes de tipo locales y sistémicos ${ }^{(1)}$. Dentro de los factores locales, los de origen mecánico-traumático se relevan especialmente por el uso continuo de prótesis removible, que produce lesiones microtraumáticas, disminuye flujo y $\mathrm{pH}$ salival, dificulta la llegada de anticuerpos salivales y el barrido mecánico lingual, favoreciendo la aparición de un microambiente ácido y anaerobio que permite proliferación de bacterias y hongos oportunistas. En relación al factor higiénico-infeccioso, una mala higiene oral y protésica permite rápido desarrollo y acúmulo de biofilm sobre la superficie protésica produciéndose adhesión de especies como Staphylococcus aureus, Streptococcus mutans y Lactobacillus y microorganismos del género Candida, aumentando el riesgo de $\operatorname{ESP}^{(2,3,4)}$.

Una vez establecido el cuadro de ESP, la aparición de signos y síntomas clínicos se correlaciona fuertemente con recuentos de levaduras superiores a $400 \mathrm{UFC} / \mathrm{ml}$ de saliva ${ }^{(5)}$. Aunque existen sujetos con niveles altos de Candida sin signos clínicos y sujetos con recuentos bajos que $\mathrm{s}$ padecen la infección, lo cual indica el rol de otros factores locales y/o sistémicos involucrados en la etiopatogenia ${ }^{(2,6)}$. Sin embargo, de los sujetos con ESP, el $71,4 \%$ son portadores de Candida albicans y de acuerdo a estudios de nuestro equipo ${ }^{(7)}$ y otros, la severidad de ESP se relaciona directamente con la presencia de esta especie de levadura en el $77,4 \%$ de los $\operatorname{casos}^{(8)}$.

El tratamiento de ESP asociada a Candida es complejo, requiriéndose identificar, corregir o eliminar factores predisponentes locales y sistémi$\cos ^{(9)}$.

En una revisión reciente de la literatura sobre el tratamiento de la Candidiasis Oral ${ }^{(10)}$, se analizan 24 artículos sobre terapia antifúngica de esta condición. Los agentes farmacológicos incluidos en estos estudios fueron itraconazol, miconazol, ketoconazol, fluconazol, clotrimazol, ariconazol, amfotericina b y nistatina.

En relación a la efectividad de miconazol gel en el tratamiento de ESP, un estudio reporta una efectividad del $80 \%(11)$, el estudio de Vasconcelos et al., ${ }^{(12)}$ describe una efectividad del $90 \%$, mientras que la mayoría de los estudios han descrito una efectividad en casi el $100 \%$ de los $\operatorname{casos}^{(13,14,15,16)}$. Por estas razones el tratamiento tópico con gel de miconazol es el de elección en casos de ESP. Desde el punto de vista farmacológico, los antifúngicos poliénicos e imidazoles, han demostrado inhibir el crecimiento de Candida albicans, con remisión de signos y síntomas luego de 12 a 14 días de tratamiento(17). Sin embargo, se describe que algunas cepas de levaduras del género Candida desarrollan resistencia a ellos ${ }^{(10)}$.

El propósito de este estudio fue determinar el recuento salival de levaduras del género Candida en adultos mayores portadores de prótesis removible con candidiasis oral asociada a ESP, antes y después de ser tratados con miconazol. Los resultados de este estudio podrían ampliar el conocimiento de la efectividad de este antifúngico en el tratamiento de ESP, pudiendo ser un aporte en la determinación de nuevas terapias antifúngicas en esta patología.

\section{MATERIALES Y MÉTODOS}

Tipo de estudio. Transversal descriptivo. Se incluyeron 32 sujetos mayores de 60 años de ambos géneros con diagnóstico de ESP, reclutados en el Servicio de Diagnóstico de la Facultad de Odontología de la Universidad de Chile o en el Centro de Referencia de Salud, Peñalolén Cordillera Oriente. Se excluyeron sujetos adultos mayores con enfermedades de base no controladas, sujetos con alergia a miconazol y sujetos con deterioro cognitivo diagnosticado. Se corroboró que no hubiesen estado bajo tratamiento antimicrobiano por al menos 3 meses antes de estudio. El estudio fue aprobado por el Comité de Ética del Servicio de Salud Metropolitano Oriente y fue realizado conforme a la declaración de Helsinki(18). Los sujetos firmaron un consentimiento informado de su participación en el estudio. Un equipo de odontólogos calibrados, realizó anamnesis y examen para determinar la presencia de candidiasis oral subprotésica basado en los signos clínicos. La ESP se clasificó según severidad en: Tipo I: lesión inflamatoria simple y localizada; Tipo II: lesión inflamatoria simple generalizada; Tipo III: lesión inflamatoria crónica con hiperplasia papilar granulomatosa ${ }^{(19)}$.

A cada sujeto del estudio se le entregó un tubo con 40 gr de miconazol al $2 \%$ en plastibase, preparado por recetario magistral. Todos los individuos aplicaron un $\mathrm{cm}$. de antifúngico sobre la prótesis dental, 4 veces al día, durante dos semanas. Se realizó evaluación clínica y se tomó muestra de saliva para determinar el recuento de colonias del género Candida al inicio, al día 8 y al día 15 del estudio.
Toma de muestra. En el día de la visita, los sujetos estaban en ayunas mínimo $2 \mathrm{~h}$, no fumaron, ni realizaron procedimientos de higiene oral previo a la toma de muestra. Se depositaron $2 \mathrm{ml}$ de saliva en un frasco plástico estéril y las muestras se trasladaron refrigeradas, al Laboratorio de Bioquímica y Biología Oral de la Facultad de Odontología de la Universidad de Chile, siendo procesadas en menos de $4 \mathrm{~h}$.

Procesamiento de las muestras y recuento de levaduras del género Candida. Para obtener la cantidad de unidades formadoras de colonias (UFC) se realizó el método de recuento viable en medio selectivo sólido (placa agar). Las muestras de saliva se homogeneizaron y diluyeron en PBS. Se sembraron $100 \mu$ de diluciones de saliva en duplicado, en medio Sabouraud sólido suplementado con Cloranfenicol (CAF) $(50 \mu \mathrm{g} / \mathrm{ml})$. Las placas se incubaron a $30^{\circ} \mathrm{C}$ por $48 \mathrm{~h}$ en condiciones de aerobiosis y se contabilizaron las colonias desarrolladas en las placas compatibles con levaduras del género Candida, a las que se les realizó frotis y tinción de Gram. Se observaron al microscopio de transmisión (Carl Zeiss, Axiostar plus) para confirmar que las colonias eran compatibles con levaduras.

Análisis Estadístico. Debido a la distribución de los datos, los valores de edad y recuento de levaduras se expresaron en mediana y rangos. Los datos clínicos se expresaron en frecuencia y porcentaje. Para la asociación entre variables se utilizó el test de Mann-Whitney y el test de Chi-Cuadrado Para el análisis comparativo del cambio en los recuentos de colonias de levaduras en los días 0,8 y 15 del tratamiento, se utilizó la prueba de Kruskal-Wallis. Se analizaron los datos utilizando el software STATA versión 11.0. Se aceptaron diferencias estadísticamente significativas con un error alfa igual o menor a $0,05 \%$ y un intervalo de confianza del $95 \%$.

\section{RESULTADOS}

Análisis de datos demográficos. El total de la muestra comprendió 32 adultos mayores con candidiasis oral asociada a ESP, con mediana de edad de 68 años (rango 60-92 años). El 71,9\% $(n=23)$ de los individuos eran mujeres y el $28,1 \%(n=9)$ eran hombres. No hubo diferencias estadísticamente significativas en la edad entre hombres y mujeres.

Análisis de datos clínicos. De acuedo a la severidad de ESP, el $25 \%$ $(n=8)$, el $56,3 \%(n=18)$ y el $18,7 \%(n=6)$ de los individuos presentó ESP tipo I, II y III, respectivamente.

En el examen clínico se observó que el $18,8 \%$ de los individuos $(n=6)$ presentó queilitis angular, el 12,5\% ( $n=4)$ hiperplasia irritativa, el $6,3 \%$ $(n=2)$ úlcera traumática o eritoplasia y el $3,1 \%(n=1)$ liquen plano.

Tabla 1. Frecuencia y porcentaje de sujetos con patologías sistémicas en la muestra estudiada.

\begin{tabular}{lccc}
\hline \multicolumn{1}{c|}{ Patología } & $\mathbf{n}$ & $\%$ \\
\hline Hipertensión arterial & 20 & 62,5 \\
\hline Diabetes & 9 & 28,1 \\
\hline Artritis reumatoide & 7 & 22,6 \\
\hline Hipercolesterolemia & 4 & 12,5 \\
\hline Patología respiratoria crónica & 3 & 9,4 \\
\hline Cardiopatías & 3 & 9,4 \\
\hline Depresión & 2 & 6,3 \\
\hline Osteoporosis & 1 & 3,1 \\
\hline
\end{tabular}

En la tabla $n^{\circ} 1$ se observan las patologías sistémicas que presentaban los individuos de la muestra, siendo las más frecuentes la hipertensión $(62,5 \%)$, diabetes $(28,1 \%)$ y artritis reumatoidea $(22,6 \%)$.

Recuentos de levaduras del género Candida. Al inicio del estudio el $100 \%$ de los individuos presentaba valores de recuento de levaduras del género Candida superiores a $400 \mathrm{UFC} / \mathrm{ml}$ de saliva (Tabla 2). En esta misma tabla se puede observar que, en comparación con los valores del inicio del estudio, el recuento de levaduras disminuyó significativamente en los días 8 y 15 (test de Kruskal Wallis $p=0,001$ ), aunque en ambos tiempos se evidenció persistencia de levaduras luego de la aplicación del antifúngico (rangos $0-13.500$ y $0-20.800$, respectivamente). No 
Tabla 2. Recuentos de levaduras del género Candida observados en los individuos del estudio en los tiempos 1, 8 y 15 días.

\begin{tabular}{c|c|c|c}
\hline Tiempo & $\begin{array}{c}\text { UFC/ml saliva } \\
\text { mediana (rango) }\end{array}$ & p value & p value \\
\cline { 1 - 3 } Día 1 & $6.800(420-510.000)$ & $0,001^{* a}$ & \multirow{2}{*}{$0,001^{\&}$} \\
\cline { 1 - 3 } Día 8 & $163(0-13.500)$ & $0,001^{*}$ & $0,001^{\text {a }}$
\end{tabular}

* a Test Mann-Whitney: * Diferencias estadísticamente significativas entre dias 1 y 8 , ${ }^{a}$ Diferencias estadísticamente significativas entre dias 1 y 15.

\& Test Kruskal Wallis: Diferencias estadísticamente significativas entre los grupos.

hubo diferencias estadísticamente significativas al comparar los valores de recuento de levaduras entre los días 8 y 15 del estudio (Test Mann Whitney, $p=0,343$ ).

Con la finalidad de discriminar en qué individuos del estudio se presentaba persistencia de levaduras luego del tratamiento, procedimos a categorizar en forma arbitraria y ascendente la variable recuento de levaduras en 4 niveles de carga de levaduras (Tabla 3). En el día 1 del estudio, los 32 individuos fueron clasificados en el nivel más alto de carga de levaduras. Al día 8 y 15 del estudio el $34,4 \%(n=11)$ y el $43,8(n=14)$ de los individuos no presentaron levaduras del género Candida en saliva, respectivamente. En consecuencia, al día 8 y 15 del estudio el $65,6 \%(n=21)$ y el $56,2(n=18)$ de los individuos presentaron persistencia de levaduras del género Candida después del tratamiento con antifúngico, respectivamente (Tabla 3 ). En la Tabla 3 se encuentra desagregada, según los distintos niveles de carga de levaduras, la frecuencia de individuos portadores de levaduras después del tratamiento. Llama la atención la frecuencia de individuos con niveles superiores a $400 \mathrm{UFC} / \mathrm{ml}$ de saliva en los días 8 y 15. Independientemente de la persistencia de levaduras del género Candida después del tratamiento, en la totalidad de los individuos desaparecieron los signos clínicos de ESP.

No hubo asociaciones estadísticamente significativas entre los recuentos o cargas de levaduras post tratamiento, con patologías sistémicas, severidad de ESP o condiciones intraorales.

Tabla 3. Frecuencia de individuos y distribución de recuentos de levaduras de acuerdo a la carga de levaduras del género Candida presente en los distintos tiempos del estudio

\begin{tabular}{|c|c|c|c|c|c|c|}
\hline \multirow{2}{*}{$\begin{array}{l}\text { Carga de } \\
\text { levaduras }\end{array}$} & \multicolumn{6}{|c|}{ Tiempo de aplicación del antifúngico } \\
\hline & & Día 1 & & Día 8 & & Día 15 \\
\hline $\mathrm{UFC} / \mathrm{ml}$ & $n(\%)$ & me (rango) & $\mathrm{n}(\%)$ & me (rango) & $n(\%)$ & me (rango) \\
\hline $0-0$ & $\begin{array}{c}0 \\
(0)\end{array}$ & $0(0)$ & $\begin{array}{c}11 \\
(34,4)\end{array}$ & $\begin{array}{c}0 \\
(0)\end{array}$ & $\begin{array}{c}14 \\
(43,8)\end{array}$ & $0(0)$ \\
\hline $1-100$ & $\begin{array}{c}0 \\
(0)\end{array}$ & $0(0)$ & $3(9,3)$ & $\begin{array}{c}90 \\
(60-100)\end{array}$ & $\begin{array}{c}6 \\
(18,8)\end{array}$ & $\begin{array}{c}60 \\
(30-100)\end{array}$ \\
\hline $101-400$ & $\begin{array}{c}0 \\
(0)\end{array}$ & $0(0)$ & $\begin{array}{c}7 \\
(21,9)\end{array}$ & $\begin{array}{c}198 \\
(110-355)\end{array}$ & $\begin{array}{c}5 \\
(15,6)\end{array}$ & $\begin{array}{c}220 \\
(160-355)\end{array}$ \\
\hline$>400$ & $\begin{array}{c}32 \\
(100)\end{array}$ & $\begin{array}{c}6.085 \\
(420-510.000)\end{array}$ & $\begin{array}{c}11 \\
(34,4)\end{array}$ & $\begin{array}{c}1.370 \\
(440-13.500)\end{array}$ & $\begin{array}{c}7 \\
(21,8)\end{array}$ & $\begin{array}{c}2.230 \\
(685-20.800)\end{array}$ \\
\hline
\end{tabular}

Carga de levaduras $=$ Categorización arbitraria ascendente de los valores de recuentos de levaduras

\section{DISCUSIÓN}

Se sabe que con el envejecimiento, el epitelio oral se atrofia y la síntesis de colágeno disminuye, favoreciendo la prevalencia de lesiones de la mucosa oral. En Chile, dicha prevalencia en adultos mayores ha sido poco estudiada. En 2003, un estudio transversal conducido por nuestro equipo de investigadores ${ }^{(20)}$, determinó una prevalencia del $53 \%$ de una o más lesiones de la mucosa oral, siendo la más prevalente la estomatitis protésica (ESP), que afecta al $34 \%$ de sujetos portadores de prótesis removible (PR). Estos datos han sido corroborados en un estudio más reciente, en donde se describe una prevalencia de un $67,5 \%$ de lesiones de la mucosa oral y un $37,1 \%$ de $\mathrm{ESP}^{(21)}$.

Chile se encuentra en una transición demográfico-epidemiológica hacia el envejecimiento poblacional. Respecto de los problemas dentales, la encuesta nacional de salud 2009-2010(22), devela el estado de salud oral de la población de 65 años y más, señalando que alrededor del 75\% son desdentados parciales (promedio de 7 dientes remanentes), de los cuales $37,1 \%$ porta prótesis removible en ambos maxilares, $25,3 \%$ en el maxilar superior y $0,8 \%$ en el inferior. De acuerdo a este panorama, es que se ha establecido como objetivo estratégico en salud "la protección y recuperación de la salud buco-dental del adulto de 60 años".

De acuerdo a la guía clínica del MINSAL "Salud oral integral para adultos de 60 años"(23), se postula que ESP se debe tratar como candidiasis y que se pueden indicar antimicóticos tópicos específicos, ya sea de la familia de polienos (nistatina, amfotericina B) o de los azoles (miconazol, clotrimazol, ketoconazol, itraconazol y fluconazol), alguno de los cuales se pueden usar como enjuagues bucales o pastillas que generalmente se administran por 5 a 10 días.

El propósito de este estudio fue determinar el recuento salival de levaduras del género Candida en adultos mayores portadores de prótesis removible con candidiasis oral asociada a ESP, antes y después de ser tratados con miconazol, con la finalidad de obtener evidencia sobre la efectividad de este antifúngico en el tratamiento de esta patología.

Aunque este estudio incluyó una muestra relativamente pequeña $(n=32)$, es relevante considerar que el $56,2 \%$ de los individuos presentó persistencia de levaduras del género Candida después del tratamiento tópico con miconazol al $2 \%$ en plastibase durante 14 días. Considerando que una vez establecido el cuadro, la aparición de signos y síntomas clínicos de candidiasis oral se correlaciona fuertemente con recuentos de levaduras superiores a $400 \mathrm{UFC} / \mathrm{ml}$ de saliva, es particularmente significativo el hecho de que el $21,8 \%$ de los individuos presentara recuentos de levaduras superiores a este valor, después del tratamiento. Sin embargo, en la totalidad de los individuos del estudio desaparecieron los signos clínicos de ESP, a pesar de la persistencia de altos recuentos de levaduras en saliva. Estudios adicionales se hacen necesarios para determinar la posibilidad de reaparición de ESP en estos individuos.

Adicionalmente, a pesar de la efectividad demostrada de miconazol gel en el tratamiento de ESP, este fármaco es absorbido a nivel intestinal y puede generar sensación de náuseas, irritación y diarrea ${ }^{(10)}$ además de tener un efecto en el clearance de algunas drogas anticoagulantes ${ }^{(24)}$. Además, este fármaco interfiere también las rutas metabólicas de células humanas aumentando la toxicidad, especialmente a nivel hepático( ${ }^{(25)}$. Debido a estos antecedentes, estos antifúngicos debieran reservarse para infecciones en sujetos debilitados o inmunodeprimidos. Si además consideramos que miconazol no elimina levaduras del género Candida en todos los individuos tratados, se hace necesario la realización de estudios posteriores para determinar la posible resistencia de Candida a este fármaco.

\section{FINANCIAMENTO}

Proyecto FONIS-Ministerio de Salud-CONICYT: SA15I20030

\section{CONFLICTO DE INTERÉS}

Los autores declaran no tener ningún conflicto de intereses. 


\section{Bibliografía}

1 Emami E, Taraf H, de Grandmont P, Gauthier G, de Koninck L, Lamarche C, et al. The association of denture stomatitis and partial removable dental prostheses: a systematic review. Int J Prosthodont. 2012;25(2):113-9.

2. Shimizu C, Kuriyama T, Williams DW, Karasawa T, Inoue K, Nakagawa K, et al. Association of oral yeast carriage with specific host factors and altered mouth sensation. Oral Surg Oral Med Oral Pathol Oral Radiol Endod. 2008;105(4):445-51

3. Gendreau L, Loewy ZG. Epidemiology and etiology of denture stomatitis. J Prosthodont. 2001;20(4):251-260.

4. Hoshi N, Mori H, Taguchi H, Taniguchi M, Aoki H, Sawada T, et al. Management of oral candidiasis in denture wearers. J Prosthodont Res. 2011:55(1):48-52.

5. Epstein J, Pearsall N, Truelove E. Quantitative relationships between Candida albicans in saliva and the clinical status of human subjects. J Clin Microbiol. 1980;12:475-6.

6. Murray P, Rosenthal K, Pfaller M. Microbiología médica. 6a ed. Elsevier. 2009. p. 679- 791.

7. Lee X, Gómez L, Vergara C, Astorga E, Cajas N, Ivankovic M. Association between presence of Candida yeasts and elderly patient factors with and without denture stomatitis. Int. J. Odontostomat. 2013;7(2):279-285

8. Felton D, Cooper L, Duqum I, Minsley G, Guckes A, Haug S, et al. Evidence-based guidelines for the care and maintenance of complete dentures: a publication of the American College of Prosthodontists. J Prosthodont. 2011;20(Suppl 1):S1-S12.

9. Salerno C, Pascale M, Contaldo M, Esposito V, Busciolano M, Milillo L, et al. Candidaassociated denture stomatitis. Med Oral Patol Oral Cir Bucal. 2011;16:39-143.

10. Garcia-Cuesta C, Sarrion-Pérez MG, Bagán JV. Current treatment of oral candidiasis: A literature review. J Clin Exp Dent. 2014;6(5):e576-582

11. Capistrano HM, de Assis EM, Leal RM, Alvarez-Leite ME, Brener S, Bastos EM. Brazilian green propolis compared to miconazole gel in the treatment of Candidaassociated denture stomatitis. Evid Based Complement Alternat Med. 2013:947980. 12. Vasconcelos LC, Sampaio MC, Sampaio FC, Higino JS. Use of Punica granatum as an antifungal agent against candidosis associated with denture stomatitis. Mycoses. 2003 Jun;46(5-6):192-6.

13. Santos VR, Gomes RT, de Mesquita RA, de Moura MD, França EC, de Aguiar $E G$, et al. Efficacy of Brazilian propolis gel for the management of denture stomatitis: a pilot study. Phytother Res. 2008;22(11):1544-1547.
14. Amanlou M, Beitollahi JM, Abdollahzadeh S, Tohidast-Ekrad Z. Miconazole gel compared with Zataria multiflora Boiss gel in the treatment of denture stomatitis. Phytother Res. 2006;20(11):966-969.

15. Pinelli LA, Montandon AA, Corbi SC, Moraes TA, Fais LM. Ricinus communis treatment of denture stomatitis in institutionalised elderly. $\mathrm{J}$ Oral Rehabil. 2013:40(5):375-380.

16. Tay LY, Jorge JH, Herrera DR, Campanha NH, Gomes BP, Andre Dos Santos $\mathrm{F}$. Evaluation of different treatment methods against denture stomatitis: a randomized clinical study. Oral Surg Oral Med Oral Pathol Oral Radiol. 2014;118(1):72-77.

17. Lamfon H, Porter SR, McCullough M, Pratten J. Susceptibility of Candida albicans biofilms grown in a constant depth film fermentor to chlorhexidine, fluconazole and miconazole: a longitudinal study. J Antimicrob Chemother. 2004;53(2):383-385.

18. Wilson CB. An updated Declaration of Helsinki will provide more protection. Nat Med. 2013;19(6):664.

19. Koeck B. Prótesis Completas. 4a ed. Urban \& Fischer. 2007. p. 344-346.

20. Espinoza I, Rojas R, Aranda W, Gamonal J. Prevalence of oral mucosal lesions in elderly people in Santiago, Chile. J Oral Pathol Med. 2003;32:571-575.

21. Cueto A, Martínez R, Niklander S, Deichler J, Barraza A, Esguep A. Prevalence of oral mucosal lesions in an elderly population in the city of Valparaiso, Chile. Gerodontology. 2013;30(3):201-206

22. ENS 2010: Encuesta Nacional de Salud 2009-2010, Ministerio de Salud Chile. http://www.redsalud.gov.cl/portal/url/item/99bbf09a908d3eb8e04001011f014b49. pdf

23. MINISTERIO DE SALUD. Guía Clínica Salud Oral Integral para Adultos de 60 años. 2007. Santiago: Minsal.

24. De Pauw A, De Backer T. Miconazole buccal gel and risk for systemic bleeding how certain topical formula can interfere with anticoagulants. Acta Clin Belg. 2015;70(2):121-123.

25. Williams D, Kuriyama T, Silva S, Malic S, Lewis MA. Candida biofilms and oral candidosis: treatment and prevention. Periodontol 2000. 2011;55:250-265. 